\title{
Melatonin and clinical application
}

\author{
Lennart Wetterberg
}

Department of Clinical Neuroscience, Karolinska Institute, Stockholm, Sweden

(Received 25 March 1999; accepted 16 April 1999)

\begin{abstract}
A review of the different publications dealing with melatonin in humans shows that this field has been very active in the last few years. Normative melatonin values have been defined. Various relationships between melatonin and other traits have been studied, such as sleep, circadian rhythm, surgical stress and anaesthesia. Age-related melatonin studies and melatonin during depression and other psychiatric disorders have been reviewed. Finally, some studies have been performed to use melatonin as a medication for sleep disturbance in depression, for jet-lag and as a skin protector for ultraviolet light. (C) Inra/Elsevier, Paris
\end{abstract}

melatonin / clinics / depression / sleep / circadian

Résumé - Mélatonine et applications cliniques. Une revue des différentes publications traitant de la mélatonine chez l'homme montre que ce champ a été très actif au cours de ces dernières années. Des normes de concentrations de mélatonine ont été définies. Diverses relations entre la mélatonine et d'autres caractéristiques comme le sommeil, les rythmes circadiens, le stress chirurgical et l'anesthésie ont été étudiées. Des études sur la mélatonine au cours du vieillissement et sur la mélatonine pendant la dépression et au cours d'autres désordres psychiatriques ont été conduites. Enfin, plusieurs essais ont utilisé la mélatonine comme médicament pour traiter les troubles du sommeil pendant la dépression, pour atténuer le jet-lag et comme protecteur de la peau contre les UV. (C) Inra/ Elsevier, Paris

\section{mélatonine / clinique / dépression / sommeil / circadien}

\section{INTRODUCTION}

Over the last couple of years there has been a rapid development in studies about the pineal hormone melatonin in humans. The situation today is very different from two decades ago when only a few clinical results for melatonin were available. An update of the clinical application of melatonin was reviewed in the progress report by Webb et al. [64] following the pineal conference 1996 in Sitges, Spain.

\footnotetext{
* Correspondence and reprints: Psychiatric Research Center for Finnmark and Troms Counties, N-9291 Troms $\emptyset$, Norway

E-mail: Lennart.Wetterberg@rito.no
} 
In the present review 70 references about melatonin have been selected from more than 1000 publications that have appeared in the past 3 years. This summary is thus limited to a few studies and focuses mainly on new and often complex results about melatonin in humans. It is still too early to try to summarize the knowledge regarding melatonin in humans into a comprehensive hypothesis about its action. However, the methodology in human studies has become more and more sophisticated as the research front moves forward. The relative importance of the different studies is not possible to judge at this point.

Several excellent books and review articles about melatonin have also been published in the last few years. Bergstrom and Hakanson [7] reviewed the history of the pineal gland from 2300 years ago. Lerner and associates discovered melatonin in 1958. Also included in their review was the 'melatonin hypothesis' of Wurtman and Axelrod in 1965 , according to which the pineal gland acts as a transducer responding to changes in light by changing its rates of melatonin output.

Clinical applications in psychiatry and neoplastic disorders have been reviewed by Shafii and Shafii [48] and the possible relationship between breast cancer and melatonin was published in a book by Stevens et al. [50] and in a review by Brainard et al. [8]. Continued research into magnetic field exposure shows that this may reduce melatonin concentrations in humans [21].

Touitou [57] has pointed out that the secretion and release of melatonin depend upon a large number of exogenous and endogenous factors such as sex, age, pubertal stage, menstrual cycle, drugs, season and light. It is also a time-clue provider used by humans to adapt to the environment.

The pineal and psychiatry was reviewed by Mullen and Silman [36]. Modern research is now demonstrating the pineal to be an endocrine gland capable of affecting the brain and behaviour. Present ideas about melatonin and psychiatry suggest a positive involvement of melatonin in affective disorders, possible involvement in the schizophrenic psychosis and potential involvement of this hormone in other psychiatric categories. In one review Miles and Philbrick [33] discuss the etiological significance, diagnostic potential and clinical utility of melatonin in psychiatry.

Kendler [22] has recently given an overview of the claims for melatonin as a potential treatment in many conditions where reductions in melatonin secretion have been associated, e.g. cardiovascular disease, Alzheimer's, diabetes, SIDS and ageing. He states "however, melatonin's role in their aetiology and/or pathophysiology is unproven". A review of further prospects of the possible clinical utilization of melatonin by Bubenik et al. [10] covers a wide range of diagnoses and conditions.

As a basis for human studies a worldwide reference data bank of melatonin excretion across different ages and latitudes has recently been reported by Wetterberg et al. [66]. The study of individuals at 19 geographic locations provides an opportunity to obtain a global estimate of normative nighttime urinary melatonin concentration and its relationship with various parameters, i.e. gender, age range (18-62 years), height, weight, urine volume and geographic location.

\section{MELATONIN RECEPTORS AND RECEPTOR FUNCTIONS}

The development of characterizing melatonin receptor subtypes is rapid. Recent efforts by Gubitz and Reppert to clone further members of the melatonin receptor family have led to the identification of a novel G-protein-coupled receptor in human pituitary [18]. The gene was found to be $\mathrm{X}$-linked. Radiation hybrid mapping revealed that the gene (GPR50) is localized to $\mathrm{Xq} 28$. This locus might be relevant to genetically based neuroendocrine disorders in humans. 
Fraschini's group [38] has reported on a method for the evaluation of drug action at the human melatonin receptor subtypes and showed for the first time differences in intrinsic activity between human MT1 and MT2 receptors. Al-Ghoul et al. [1] reported a distinct cellular distribution in the human cerebellar cortex of the mammalian Mella and Mel1b melatonin receptor subtypes. They demonstrated that Mellb melatonin receptor mRNA was expressed in human cerebellar Bergmann glia and astrocytes. The Mella melatonin receptor mRNA was expressed in both basket-stellate cells and granule cells. They concluded that the melatonin receptors are heterogeneously expressed in human cerebellar cortex.

Many functional studies show that melatonin plays a fundamental role in neuroimmuno modulation. Involvement of nuclear binding sites for melatonin in the regulation of IL- 2 and IL- 6 production by human blood mononuclear cells has been reported by Garcia-Maurino et al. [17]. Their results in monocyte cultures resembled closely those shown in human peripheral blood mononuclear cells and confirm the involvement of a nuclear mechanism in the melatonin effects on cytokine production in human mononuclear cells. A recent study by Fjaerli et al. [16] indicate that melatonin may amplify cellular activation reactions that are protein kinase $\mathrm{C}$ dependent. This mechanism may have physiological importance in upregulation of the immune system.

Elevated nocturnal melatonin may be a consequence of gonadotropin-releasing hormone (GnRH) deficiency in women with hypothalamic amenorrhea. Kadva et al. [20] found elevated nocturnal melatonin in women with idiopathic hypogonadotropic hypogonadism $(\mathrm{IHH})$. Melatonin levels were elevated in six of seven patients compared with their matched controls. The finding of elevated nocturnal melatonin in GnRH-deficient women with IHH suggests that nocturnal melatonin is elevated as a consequence of GnRH deficiency, irrespective of its etiology.

\section{SLEEP STUDIES AND MELATONIN}

The pineal hormone melatonin is considered to play a major role in the circadian regulation of sleep. Kunz et al. [23] studied pineal calcification and its relation to subjective sleep perception. They classified the degree of pineal calcification (DOC) into seven groups using cranial computer tomography (CT) and then correlated pineal DOC to chronic subjective sleep-related disturbances as measured by a sleep questionnaire in 36 patients. Analysed by logistic regression models higher pineal DOC was significantly associated with the presence of daytime tiredness and sleep disturbance. This study provides initial confirmation of the hypothesis that the increasing degree of pineal calcification (DOC) might indicate a decrease in melatonin production, which consecutively might lead to a disturbed circadian rhythmicity in the sleep-wake cycle, with the principal symptom being daytime tiredness.

Altered circadian melatonin secretion patterns in relation to sleep in patients with chronic sleep-wake rhythm disorders has been studied by Rodenbeck et al. [47]. They examined the circadian rhythm of circulating melatonin and the sleep-wake rhythm in five patients with chronic sleep-wake rhythm disorders and ten age-matched healthy controls. All patients showed altered circadian melatonin rhythm parameters in relation to their sleep-wake cycle compared to age-matched controls. These alterations were random, i.e. independent of the type, the duration and the age of onset of the disorder. The melatonin onset to sleep onset interval varied between the patients and the melatonin acrophase to sleep offset interval was prolonged in four patients. These findings indicate individual phase relations between the circadian melatonin rhythm and the sleep-wake cycle in patients with chronic sleep-wake rhythm disorders. Since the prolonged melatonin acrophase to sleep offset interval was the most consistent find- 
ing independent of aetiological origins, this abnormality may be one possible maintaining factor in chronic sleep-wake rhythm disorders due to reduced phase-resetting properties of the circadian pacemaker. The authors hypothesize that low circadian melatonin amplitudes and a subsensitivity to daylight maintain the disorder in at least some patients.

In this context a study of evening administration of melatonin and bright light and the interactions on the EEG during sleep and wakefulness is of interest. Cajochen et al. [11] used a placebo-controlled balanced cross-over design. They investigated the acute effects of exogenous melatonin, $5 \mathrm{mg}$ per os at 20.40 hours, with or without a 3-h bright light exposure with 5000 lux from 21.00-24.00 hours on subjective sleepiness, internal sleep structure and EEG power density during sleep and wakefulness in healthy young men. The acute effects of melatonin, bright light and their interaction were measured on the first treatment day and possible circadian phase shifts were assessed on the post-treatment day. On the treatment day, the evening rise in subjective sleepiness was accelerated after melatonin and protracted during bright light exposure. Melatonin shortened and bright light increased sleep latency. On the post-treatment day after evening bright light, the rise in sleepiness and the onset of sleep were delayed, independently of whether melatonin was coadministered or not. Thus, although acute bright light and melatonin administration affected subjective sleepiness, internal sleep structure and EEG power density during sleep and wakefulness in an additive manner, the phase-shifting effect of a single evening bright light exposure could not be blocked by exogenous melatonin.

Melatonin effects on sleep, mood and cognition in elderly with mild cognitive impairment were studied by Jean-Louis et al. [19]. The effects of immediate-release melatonin on circadian rest-activity profiles, $\operatorname{cog}$ nition and mood were investigated in ten elderly individuals with self-reported sleep-wake disturbances. Melatonin, $6 \mathrm{mg}$, administered $2 \mathrm{~h}$ before habitual bedtime, enhanced the rest--activity rhythm and improved sleep quality as observed in a reduction in sleep onset latency and in the number of transitions from sleep to wakefulness. However, total sleep time was not significantly increased nor was wake within sleep significantly reduced. The ability to remember previously learned items improved along with a significant reduction in depressed moods. No side effects or contraindications were reported by any of the participants during the 10-day trials. These data suggest that melatonin can safely improve some aspects of sleep, memory and mood in the elderly in short-term use.

Although melatonin is related to sleep and sleep disturbances in many instances this is not a general rule. In patients with obstructive sleep apnea syndrome melatonin did not change with treatment. Wikner et al. [68] studied melatonin secretion and excretion in patients with obstructive sleep apnea syndrome. Nine men were tested on two occasions: immediately before initiation of continuous positive airway pressure (CPAP) treatment and again after 4 weeks of continuous nocturnal use of CPAP. Serum melatonin was determined every second hour between 20.00 and 08.00 hours. The melatonin secretion among the obstructive sleep apnea syndrome patients did not differ from that found in healthy controls although sleep apnea recordings were normalized during CPAP treatment.

\section{BIOLOGICAL RHYTHMS AND MELATONIN}

Shift type and season affect adaptation of the melatonin rhythm in offshore oil rig workers. Barnes et al. [6] have shown that the 6-sulphatoxymelatonin rhythm of oil rig workers on a 2 -week night shift (18.00-06.00 hours) adapts to the shift via a phase delay. They reported that on two offshore drill crews working a 1-week day 
(12.00-00.00 hours) and 1-week night (00.00-12.00 hours) swing shift. Urine samples were collected every $2-3 \mathrm{~h}$ throughout the subjective days, with over-sleep collections, for the measurement of 6-sulphatoxymelatonin. One crew $(n=11)$, studied in November, showed no change in their 6-sulphatoxymelatonin rhythm during the night shift. The other crew $(n=7)$, studied in March, showed a significant phase advance of the rhythm during the night shift. The data indicate that both the type of shift and the season influence the direction and degree of adaptation.

Adjustment of the human melatonin rhythm to shortening of the natural summer photoperiod was studied by VondrasovaJelinkova et al. [60]. They exposed 15 human subjects to natural outdoor summer light from 04.15 hours until 20.00 hours for 4 days and then from 08.00 hours until 16.00 hours for another 4 days. Following shortening of the natural summer photoperiod, the time of the evening melatonin rise phase advanced by about $1.5 \mathrm{~h}$ within 1 day and further did not change significantly. The study showed that the compressed melatonin rhythm waveform in humans experiencing a long natural summer photoperiod from sunrise until sunset may change rapidly following a shortening of the photoperiod.

An excellent review of the molecular rhythms in the pineal gland has recently been published by Li et al. [28]. They report the research which has clarified the mechanisms regulating the night- and pineal-specific transcription of serotonin $\mathrm{N}$-acetyltransferase. The tissue-specific gene expression within the pineal gland and retina derives, in part, from a pineal/retina-specific transcription factor, cone-rod homeobox protein, which binds to a pineal regulatory element. This regulatory element is present in promoters of pineal-selective enzymes, such as $N$-acetyltransferase, hydroxyindole- $O$-methyl transferase and pineal night-specific ATPase.

Daily and seasonal variations in the concentration of melatonin in the human pineal gland have been studied by Luboshitzky et al. [31]. They determined the concentration of melatonin in human pineal glands obtained at autopsy from 66 male subjects, aged 16-84 years over a period of 12 consecutive months. Based on the time of death, a day-night difference in pineal melatonin levels was evident only in the long photoperiod (April-September) with significantly higher melatonin concentrations occurring at night (22.00-10.00 hours). Night-time values in the long photoperiod were significantly higher than the night-time values during the short photoperiod (October-March). During the short photoperiod, the data suggested a possible phase delay in melatonin secretion. Day-night difference was evident in young subjects (30-60 years), but not in elderly subjects (61-84 years). These data indicate that the concentration of melatonin in the human pineal is augmented only during the long photoperiod. The results suggest a partial effect of photoperiod on melatonin secretion in humans. This may result from living in an artificial light environment or may be due to other non-photic signals involved in generating melatonin rhythm.

\section{LIGHT, RETINAL SENSITIVITY IN HUMANS AND THE CIRCADIAN AXIS}

Light affects the circadian axis in at least two ways. It can cause the acute suppression of pineal melatonin synthesis and/or a phase shift of the circadian oscillator. As recent evidence has suggested that extraocular light exposure may cause phase shifts of the circadian clock, Lockley et al. [30] have investigated whether suppression of melatonin can be induced by the same type of light exposure. In one study the subjects' eyes were exposed to white light, 2250 lux for 30 mins, with suppression of night-time plasma melatonin levels to about $60 \%$. In a second study, light with higher intensity, 14000 or 67500 lux for 180 mins, was 
delivered in the same manner to the popliteal region behind the subjects' knees, whilst shielding their eyes. No suppression of plasma melatonin levels was detected in any of the subjects. Thus, extraocular photoreception, if it exists in mammals, does not affect the suprachiasmatic nuclei-pineal pathway measured as suppressed plasma melatonin in humans.

Effects of indirect light and propranolol on melatonin levels in normal human subjects were studied by Mayeda et al. [32]. They developed an indirect lighting protocol to measure nocturnal melatonin suppression by light in humans, who sat with five light sources placed behind them. Lights reflecting before each subject produced light that measured 500 lux. Subjects went to bed in darkness by midnight and then sat in light from 02.00 to 04.00 hours. Blood for melatonin was drawn at $20-30$-min intervals from midnight to 06.00 hours. Plasma melatonin was measured. The indirect lighting protocol was used to compare the effects of 500 lux light to dark (21 subjects) and to study varying light intensities from 300 to 2000 lux (seven subjects). They also studied the effects of propranolol plus dark and propranolol plus 500 lux light on melatonin levels. Subjects received placebo, $10 \mathrm{mg}$ propranolol or $40 \mathrm{mg}$ propranolol orally at 23.00 hours, and were then exposed to either the dark or light condition. Melatonin levels obtained with the indirect lighting protocol were consistent with studies using direct lighting; light of 500 lux significantly suppressed nocturnal melatonin and suppression was dose-related between 300 and 2000 lux. Sitting in dim light had no significant effect on melatonin suppression. Propranolol caused a dose-dependent decrease in melatonin levels in both the dark and the light.

Aoki et al. [2] studied the minimum light intensity required to suppress nocturnal melatonin concentration. Five healthy male volunteers were exposed to light at different intensities $(<10,500,1000,2500$ and
$5000 \operatorname{lux}$ ) in a repeated-measure design. Suppression of melatonin was dependent on both light intensity and duration of light exposure. Minimum intensities of light suppressing nocturnal melatonin levels were calculated as $393,366,339$ and 285 lux for exposure durations of $30,60,90$ and 120 min, respectively. Minimum effective intensity and duration of light exposure showed a linear inverse relationship. These results suggest that a lower intensity of light than previously reported suffices to suppress melatonin in humans, and that caution is required in interpreting studies using long exposure to dim light as a background condition.

Two recent reports by Daan's [59] and Kripke's [24] groups indicate that the nocturnal suppression response to light in humans depends on the area of the retina exposed. Visser et al. [59] found that melatonin suppression by light is maximal when the nasal part of the retina is illuminated and Lasko et al. [24] suggest that light treatment of circadian phase disorders might be most effective when applied in the upper visual field. The reason for hypothesizing uneven spatial sensitivity distribution stems from animal work that revealed that retinal ganglion cells projecting to the suprachiasmatic nuclei ( $\mathrm{SCN}$ ) are unequally distributed in several species of mammals. These new interesting findings may lead to clinical improvement of the methods of light treatment in different clinical conditions and in revealing clinical subgroups of patients with different regional retinal sensitivity to light exposure.

\section{SURGICAL STRESS, ANAESTHESIA AND MELATONIN}

Biorhythms, such as regular variation in core body temperature and the pattern of the secretion of melatonin, are thought to be mediated by the same biological clock. Core body temperature is affected by the inflammatory response to major surgery. 
Apart from the well-known inhibitory effect of bright light on its secretion, melatonin is an exceedingly good marker of one of the central generating systems of circadian rhythms. Nishimura et al. [37] sequentially measured the plasma melatonin concentration pattern in patients who had undergone oesophagectomy with thoracotomy to elucidate the circadian rhythm after major surgery. From seven patients who underwent oesophagectomy with thoracotomy for oesophageal cancer, plasma concentrations of melatonin were measured. Blood samples were collected via each patient's arterial line at $00.00,02.00,04.00,06.00,08.00$, $12.00,16.00,20.00$ and 24.00 hours on the first post-operative day. Four patients out of seven had melatonin concentrations of over $30 \mathrm{pg} \cdot \mathrm{mL}^{-1}$ (mean $34 \mathrm{pg} \cdot \mathrm{mL}^{-1}$ ) at 24.00 hours on the first post-operative day. Five patients showed circadian secretion patterns of melatonin during the first postoperative day. Even the stress caused by extremely invasive surgery did not significantly disturb the melatonin secretion pattern.

However, the picture may be more complicated if different types of anaesthesia are studied. Reber et al. [44] reported on the effect of general anaesthesia on circulatory melatonin. The influences of general anaesthesia and surgery on circulating melatonin, prolactin and cortisol concentration were investigated in 32 female patients scheduled for elective gynaecological surgery to study differences in hormone profiles and responses during anaesthesia and the recovery period. Patients were randomly assigned to one of two groups. General anaesthesia was induced with either thiopentone/fentanyl or propofol/fentanyl. Maintenance of anaesthesia was achieved with either isoflurane/fentanyl or propofol/fentanyl. During anaesthesia, patients' eyes were carefully taped shut to prevent light effects. Isoflurane and propofol anaesthesia as well as darkness elicited elevated plasma melatonin levels that persisted in the recovery period in patients anaesthetized with isoflurane, but gradually decreased during the recovery of patients anaesthetized with propofol. The authors concluded that higher plasma levels of melatonin during the recovery period following isoflurane anaesthesia may explain increased sedation in these patients compared with patients who received propofol anaesthesia.

The two studies with surgical/anaesthesia stress show that the relationship between stress and melatonin levels may not be simple and straightforward and that several factors influence regulation of melatonin.

\section{AGE-RELATED MELATONIN STUDIES}

\subsection{Melatonin in the human foetus}

The role of melatonin in the human foetus has been reviewed by Thomas et al. [56]. Maternal melatonin crosses the placenta and enters the foetal circulation. Its influence may begin in utero. Melatonin receptors are widespread in the human foetus and occur in both central and peripheral tissue from early in foetal development. Thus, the influence of melatonin on the developing human foetus may not be limited to entraining circadian rhythmicity. The authors state that the transplacental availability of melatonin to the foetus should limit intake of melatonin by pregnant women. Similar warnings are given by Ciesla [12] who finds that high maternal melatonin concentrations may be responsible for inducing foetal pathologies. Her hypothesis proposes a mechanism of melatonin action, which may explain the role of this neurohormone in human pathologies, including foetal abnormalities. The hypothesis suggests that melatonin, by influencing the concentration of $\mathrm{ACTH}$ and betaendorphin and their relative proportions, could determine the stimulation or suppression of the immune system, thereby confirming its role as an immunomodulator. According to the proposed hypothesis, the risk of bearing a developmentally handi- 
capped child would be highest in a woman with a high circadian secretion of melatonin. The hypothetical break-up of maternal melatonin dimers when crossing the placenta would be the cause of an excessive concentration of melatonin monomers in the foetus, and thus it should be the reason for very low levels of vimentin filaments and cAMP concentration in embryonal cells, the latter being directly responsible for inducing foetal pathologies.

\subsection{Melatonin studies in children}

Melatonin treatment has been used in children with psychomotor and mental retardation and an irregular sleep-wake pattern. Pillar et al. [41] reported on an institutionalized 13-year-old girl with psychomotor retardation who suffered from an irregular sleep-wake pattern. Melatonin was low and without significant day-night differences. Administration of $3 \mathrm{mg}$ melatonin at night resulted in improvements in her sleep-wake pattern.

Another study in blind children with severe circadian sleep-wake disturbances shows that melatonin may help to treat sleep disorders. Palm et al. [39] used oral melatonin therapy in eight children in an open study. All patients were functionally blind and mentally retarded. Baseline sleep diaries kept by the caregivers before treatment showed non-24-h sleep-wake syndrome. Diurnal variations in serum and urinary melatonin were examined. Melatonin secretion peak time was delayed in seven patients. Body temperature variation was out of phase relative to sleep and melatonin in five patients, and thus they had signs of internal desynchronization. Melatonin given in the evening dramatically improved the sleep-wake pattern in all patients. The effect was maintained during long-term therapy for between 1 and 6 years in six patients. One patient fell back into the earlier sleep pattern after 6-8 months, and another had increasing sleep disturbance because of reflux oesophagitis, although the improvement regarding the circadian component remained. No side effects have been noted during the therapy. Oral melatonin is promising as an efficient and seemingly safe alternative for treatment of severe circadian sleep disturbances.

\subsection{Melatonin in the elderly and melatonin as an antioxidant}

Melatonin may act as a free radical scavenger and antioxidant and is believed to scavenge the highly toxic hydroxyl radical, the peroxynitrite anion, and possibly the peroxyl radical as described by Reiter [45]. It may through this action have a protective effect on oxidative damage in the central nervous system. Melatonin may also stimulate mRNA levels for superoxide dismutase and the activities of glutathione peroxidase, thereby increasing its antioxidative capacity. Also, melatonin, at least at some sites, inhibits nitric oxide synthase, a prooxidative enzyme and has been shown to reduce lipid peroxidation and oxidative damage to nuclear DNA. Melatonin has been shown prophylactically to reduce amyloid beta protein toxicity of Alzheimer's disease, to reduce oxidative damage in several models of Parkinson's disease, to protect against glutamate excitotoxicity, to reduce ischemiareperfusion injury, hyperbaric hyperoxia and a variety of neural toxins. Since endogenous melatonin levels fall markedly in advanced age, the implication of these findings is that the loss of this antioxidant may contribute to the incidence or severity of some age-associated neurodegenerative diseases.

In a further study Reiter and Maestroni [46] have studied melatonin in relation to the antioxidative defense and immune systems with possible implications for cell and organ transplantation. The age-related changes in melatonin levels in humans and its potential consequences for sleep disorders in old age was reported on by Wald- 
hauser et al. [63]. The decline of melatonin with higher age may be due to an as yet unidentified physiological mechanism accompanying senescence. The biological significance of these melatonin alterations remains unknown.

To understand the importance of melatonin in old age it is of interest to note that Liu et al. [29] found decreased melatonin levels in post-mortem cerebrospinal fluid in relation to ageing, Alzheimer's disease and apolipoprotein E genotype. Melatonin levels were determined in the cerebrospinal fluid of 85 patients with Alzheimer's disease and in 82 age-matched controls. In old control subjects over 80 years of age, CSF melatonin levels were half of those in control subjects of 41-80 years. The melatonin level in AD patients expressing apolipoprotein E-epsilon3/4 was significantly higher than that in patients expressing apolipoprotein E-epsilon4/4. In the study of Liu et al., a dramatic decrease in the CSF melatonin levels was found in old control subjects and even more so in Alzheimer's disease patients. The authors suggest that the question as to whether melatonin improves behavioural disturbances in Alzheimer's disease patients should be investigated.

In another study, a pair of monozygotic twins with Alzheimer's disease were treated with melatonin by Brusco et al. [9]. The twins had had Alzheimer's disease for 8 years when studied. The onset of the disease differed by about 6 months between the twins and was characterized by a primary impairment of memory function. Clinical evaluation at the time of diagnosis indicated a similar cognitive and neuroimaging alteration in both patients, as well as a similar neuropsychologic impairment. One of the patients was treated with melatonin in a dose of $6 \mathrm{mg}$ orally at bed time daily for 36 months. Evolution of the disease in the melatonin-treated patient indicated a milder impairment of memory function, with substantial improvement of sleep quality and reduction of sundowning. Present clinical evaluation indicated a difference in functional stage of the disease between the twins with a score of five in the twin who received melatonin and of seven in the twin who did not receive it. This is one more study in which the hypothesis that melatonin has a beneficial effect in Alzheimer's disease patients is put forward.

The seasonal inter-relationship between melatonin, vasopressin and serum osmolality in elderly subjects was studied by Asplund et al. [5]. Plasma arginine vasopressin (AVP) and melatonin and serum osmolality were measured at noon and at midnight in individuals living in the northern hemisphere in March, June, September and December, 35 healthy volunteers (15 men) aged $60-74$ years. The nocturnal increase in melatonin was highest in the autumn and lowest in the winter in both sexes. The midnight serum osmolality level was lower in the autumn than at any other time of the year. In both the men and the women the arginine vasopressin level was higher in winter than in any other season. The study suggests a possible relationship between melatonin and serum osmolality.

\subsection{The melatonin metabolite cyclic 3-hydroxymelatonin (3-OHM) - a novel biomarker}

Tan et al. [53] characterized a urinary melatonin metabolite using mass spectrometry (MS) and proton nuclear magnetic resonance as cyclic 3-hydroxymelatonin (3-OHM). Since melatonin has a wide intracellular distribution and 3-OHM is readily detected non-invasively in urine, they suggest that 3-OHM may be used to monitor in vivo $\mathrm{HO}^{*}$ levels in humans. The measurement of 3-OHM may be useful in clinical research, in the diagnosis and treatment of particularly age-related diseases.

The hypothesis that hypercalcaemia or calcium antagonism affect human melatonin secretion or renal excretion has been tested 
by Wikner et al. [69]. Patients with primary hyperparathyroidism have higher serum melatonin concentrations during active disease than after surgical cure. Whether this is caused by hypercalcaemia per se, increased parathyroid hormone secretion or other mechanisms is unknown. The authors elucidated whether exogenous hypercalcaemia influences melatonin secretion. For this purpose, eight healthy volunteers were infused with calcium and saline on separate days and in random order. Hypercalcaemia inhibited nocturnal melatonin secretion by $20 \%$ but left urinary melatonin excretion unaffected. Verapamil did not affect nocturnal melatonin secretion but increased melatonin excretion by $145 \%$. As 6-sulphatoxy-melatonin is the main melatonin metabolite excreted by the kidneys, it was tested whether verapamil would influence the excretion of 6-sulphatoxy-melatonin. In this experiment verapamil increased urinary melatonin excretion significantly (by $67 \%$ ), but left excretion of 6-sulphatoxy-melatonin unaffected. These findings imply that verapamil influences the renal and/or hepatic handling of melatonin.

\subsection{Glucose regulation and melatonin}

The role of melatonin in human glucose regulation is poorly understood. Under normal conditions, glucose tolerance is modulated by circadian rhythmicity and sleep, two central nervous system processes which may be influenced by melatonin. In the presence of a constant stimulus (e.g. intravenous glucose infusion), blood glucose levels increase from morning to evening and further increase until the middle of sleep, when a decline towards morning levels is initiated. This $24-h$ variation is due to co-ordinated changes in insulin-dependent and noninsulin-dependent glucose utilization (e.g. by the brain), in insulin sensitivity and in insulin secretion [58]. Melatonin is likely to play an indirect role in the mechanisms underlying glucose regulation via its actions on the suprachiasmatic nucleus and on sleep regulation. There is also a seasonal variation in melatonin which may modulate the glycemic response to exercise as shown by Weydahl et al. [67].

\section{MELATONIN IN DEPRESSION AND SOME OTHER PSYCHIATRIC DISORDERS}

Melatonin has been reported to be decreased in subgroups of patients with affective disorders [65]. When clusters of clinical items were correlated with the maximal nocturnal melatonin levels, significant negative regressions were found for items interpreted as retardation symptoms, especially those related to emotional or conative functions. These results point to the possibility of a 'low melatonin syndrome' in depression, characterized by low nocturnal serum levels of melatonin, an abnormal dexamethasone suppression test, a disturbed 24-h rhythm in cortisol levels and a less pronounced daily and annual cyclic variation in depressive symptomatology. Several recent studies support the hypothesis of a decreased pineal function in some types of affective disorders. Partonen [40] hypothesized that there is also a reduced production of melatonin in winter seasonal affective disorder. The reason for this would be the relative shortage of light, coincident with a predisposed metabolic error, with no switch towards serotonin synthesis among patients with winter depression.

Lewy et al. [27] have intensively studied the phase-shift hypothesis for winter depression. In a recent report they assessed different light-exposure schedules in seasonal affective disorder. Fifty-one patients and controls were studied. Subjects were exposed to bright light at either 06.00 to 08.00 hours or 19.00 to 21.00 hours for 2 weeks. Morning light phase-advanced the dim-light melatonin onset and acted more as an antidepressant than evening light, 
which phase-delayed it. This is in agreement with the report of Thalén et al. [54] who studied melatonin as a marker of circadian rhythm and the effect of bright light on melatonin in 63 depressed patients, 42 with a seasonal pattern and 21 with a non-seasonal pattern. The melatonin amplitude was significantly decreased by light, and the melatonin phase position was advanced by morning light and delayed by evening light. There was also, however, no significant difference between the seasonal and non-seasonal patients related to the degree of light suppression of melatonin and the rebound effect of serum melatonin levels following bright level exposure between 22.00 and 23.00 hours before regular light treatment.

The effect of bright light on cortisol and the relationship between melatonin and cortisol were also studied in the same 63 depressed patients [55]. Patients with a seasonal pattern improved significantly more than patients with a non-seasonal pattern of depression. A cosinor analysis showed that the cortisol batyphase was significantly advanced by morning light. The batyphase of cortisol occurred approximately $3 \mathrm{~h}$ earlier than the acrophase of melatonin. Of the changes in the melatonin acrophase $43 \%$ were reflected in a change of cortisol batyphase, indicating a hierarchical relationship with melatonin as the co-ordinating hormone transducing part of the information of the external light to the phase position of cortisol.

In other studies of depressed patients serum melatonin, platelet monoamine oxidase activity and post-dexamethasone serum cortisol predicted two main clinical subgroups as well as a subgroup of bipolar patients $[61,62]$. The identification of a patient group with latent bipolar disorder may have a potential therapeutic value since bipolar patients should be taken care of by a specialist in psychiatry, avoid tricyclic antidepressant therapy and may be candidates for lithium treatment.
Cluster headache has similar chronobiological features to periodic affective illness. The studies of cluster headache has been summarized by Costa et al. [13]. They find that cluster headache and bipolar mood disorders are conditions bearing numerous analogies, particularly as regards the temporal pattern of disturbances, the nature of predisposing or precipitating factors, the relationship with sleep, the neuroendocrine findings, and the clinical response to current treatments. Changes in the secretory pattern of melatonin have also been documented in cluster headache. Melatonin may be used in cluster headache to assess the response to pharmacological and non-pharmacological treatments.

\subsection{Fragmented melatonin rhythm in schizophrenia}

Poirier [42] has hypothesized a chronobiology disturbance of biological rhythms including melatonin in schizophrenia. The 'fragilization hypothesis' is based on the progressive development of a stress-related pathological organization in the hippocampo-hypothalamo-adrenal axis in schizophrenia. Some support to disturbed melatonin in schizophrenia is given in the study of Monteleone et al. [35] who found a decreased nocturnal secretion of melatonin in drug-free schizophrenics. They examined the circadian rhythm of plasma melatonin in nine drug-free chronic schizophrenics and in healthy subjects. The nocturnal increase in plasma melatonin levels was significantly blunted in patients as compared to controls. Chronic treatment with antipsychotic drugs significantly improved psychotic symptomatology, but did not change the secretory pattern of melatonin. These data show that the biosynthetic activity of the pineal gland is impaired in chronic schizophrenia and that successful treatment with antipsychotic drugs is not in parallel with changes in the production of melatonin. 


\subsection{Obsessive-compulsive disorder (OCD)}

Millet et al. [34] investigated the melatonin rhythm in OCD since it may provide a clue to neurotransmitter alterations, and allow a biological comparison with depression. The circadian pattern of axillary temperature was slightly different in OCD patients when compared to control subjects. No significant difference between the two groups was, however, observed for melatonin. The discrepancies with other studies may be due to the methods used, patients and control subject samples, and the biological measurement procedures.

\subsection{Fibromyalgic syndrome - a low melatonin syndrome?}

Another disease of unknown etiology is fibromyalgia which is associated with decreased nocturnal melatonin secretion [70]. Most patients with fibromyalgic syndrome complain of sleep disturbances, fatigue and pain. These symptoms might be a consequence of changed melatonin secretion. Eight patients with fibromyalgic syndrome and eight healthy controls were included in the study. The fibromyalgic syndrome patients had a $31 \%$ lower melatonin secretion than healthy subjects during the hours of darkness. This may contribute to impaired sleep at night, fatigue during the day and changed pain perception.

\subsection{Melatonin and appetite-related peptides in ageing}

There is an emerging interest in the relationship between melatonin and the appetiterelated peptides such as leptin and orexin. So far there are too few human studies to generalize the results. However, in an animal experiment it was shown that daily melatonin administration at middle age suppressed male rat visceral fat, plasma leptin and plasma insulin to youthful levels [43].
Visceral fat, insulin and leptin responses to melatonin administration may be independent of marked changes in gonadal, thyroid, adrenal or somatotropin regulation. Since increased visceral fat is associated with increased insulin resistance, diabetes and cardiovascular disease, these results may again suggest that appropriate melatonin supplementation may potentially provide prophylaxis or therapy for some prominent pathologies associated with ageing.

\section{MELATONIN AS MEDICATION}

\subsection{Treatment of sleep disturbance in depression}

There are several studies with somewhat different designs of melatonin for the treatment of sleep disturbances. In one experiment sleep problems in depressed patients has been reported by Dolberg et al. [14]. The authors' examined the hypnotic effects of slow-release melatonin during the initial 4 weeks of treatment with the serotoninuptake-blocker fluoxetine in 19 patients with major depressive disorder. Twenty-four patients with major depressive disorder were included in the study. Ten patients were treated with fluoxetine plus slow-release melatonin and nine were given fluoxetine plus placebo in a double-blind protocol for 4 weeks. Response was assessed by using rating scales for depression and sleep. Although sleep problems decreased on melatonin the hormone had no effect on the rate of improvement in symptoms of major depressive disorder. An interesting study and a review were published determining the phase-response curve of circadian rhythms to melatonin treatments $[25,26]$.

\subsection{Melatonin as treatment in jet-lag}

The efficacy of melatonin treatment in jet-lag has been reviewed by Arendt et al. [4]. The authors state that not all studies 
have been successful. Arendt [3] has discussed possible reasons for this problem and states that since melatonin has complex effects and there is a great individual sensitivity to melatonin further investigation is required not to compromise the therapeutic potential of melatonin and its agonists.

Along this research line a comparative study to determine the optimal melatonin dosage form for the alleviation of jet-lag has been performed by Suhner et al. [51]. They compared the impact of various dosage forms of melatonin and placebo on jet-lag symptoms in 320 volunteers who had flights over six to eight time zones. The volunteers received either melatonin $0.5-\mathrm{mg}$ fast-release formulation, melatonin $5-\mathrm{mg}$ fast-release formulation, melatonin 2-mg controlledrelease formulation or placebo. The study medication was taken once daily at bedtime for 4 days after an eastward flight. The fastrelease melatonin formulations were more effective than the slow-release formulation. The 5-mg fast-release formulation significantly improved the self-rated sleep quality, shortened sleep latency, and reduced fatigue and daytime sleepiness after intercontinental flight. The lower physiological dose of $0.5 \mathrm{mg}$ was almost as effective as the pharmacological dose of $5.0 \mathrm{mg}$. Only the hypnotic properties of melatonin, sleep quality and sleep latency, were significantly greater with the $5.0 \mathrm{mg}$ dose.

\subsection{Safety of melatonin}

If it turns out that melatonin is effective for jet-lag and sleep, is it safe? The impact of melatonin on driving performance was studied by Suhner et al. [52]. Twenty healthy men and women aged 21-57 years volunteered for this randomized, placebo-controlled, double-blind, crossover study. Subjective sleepiness was increased by melatonin, although the result was significant only after the prolonged concentration task. The overall result of the computer test battery showed no objective adverse impact of melatonin on driving performance. However, due to the increased subjective sleepiness after administration of this hormone, caution should be exercised when driving under the influence of melatonin. In another study Slotten and Krekling [49] tested if melatonin had an effect on cognitive performance. Using a $N=1$ double-blind alternating treatments design, daytime performance on four cognitive tests (logical reasoning, serial add/subtract, visual-spatial and four-choice reaction time tasks) was measured after per os doses of placebo and $1.6 \mathrm{mg}$ melatonin. Performance was measured during time windows corresponding to the serum melatonin peak and the temperature trough, respectively, following melatonin administration. The data showed that performance speed and accuracy measures were not affected during the serum melatonin peak, but that reaction times on all cognitive tasks increased substantially during the temperature trough. These results suggest that circulating melatonin has no direct and immediate effect on cortical information processing, and that the reduction in cognitive processing speed during body temperature trough occurs as a consequence of the hormone's hypothermic properties, causing a slowing down of cerebral processing speed.

\subsection{Melatonin as skin protector for ultraviolet radiation}

Topical melatonin was tested in combination with vitamins $\mathrm{E}$ and $\mathrm{C}$ as a skin protector from ultraviolet-induced erythema by Dreher et al. [15]. In their randomized, double-blind human study, the short-term photoprotective effects of different antioxidants and their combinations were evaluated in vivo. Vitamin $\mathrm{C}$ (ascorbic acid), vitamin $\mathrm{E}$ (alpha-tocopherol) and melatonin ( $N$-acetyl5-methoxytryptamine) were topically applied, alone or in combination, $30 \mathrm{~min}$ before ultraviolet-irradiation of the skin. The results showed a modest protective 
effect of the vitamins when applied alone and a dose-dependent photoprotective effect of melatonin. Topical application of combinations of both vitamins, or of melatonin with vitamins, enhanced the photoprotective response. Better protection was obtained by using the combination of melatonin with both vitamins. The role of reactive oxygen species and oxygen-derived free radicals, as well as potential sunscreening properties of the employed antioxidants, may explain the photoprotective effect.

\section{CONCLUSIONS}

During the last few years, the knowledge of the clinical function of melatonin and its pharmacological effects has greatly expanded. This review shows that divergent findings might equally well reflect biological as well as methodological differences. On the basis of published results about melatonin we are now able to move forward and pose new and hopefully more and more meaningful questions about its function in humans.

\section{REFERENCES}

[1] Al-Ghoul W.M., Herman M.D., Dubocovich M.L., Melatonin receptor subtype expression in human cerebellum, Neuroreport 9 (1998) 4063-4068.

[2] Aoki H., Yamada N., Ozeki Y., Yamane H., Kato N., Minimum light intensity required to suppress nocturnal melatonin concentration in human saliva, Neurosci. Lett. 252 (1998) 91-44.

[3] Arendt J., Complex effects of melatonin, Therapy 53 (1998) 479-488.

[4] Arendt J., Skene DJ., Middleton B., Lockley S.W., Deacon $S$., Efficacy of melatonin treatment in jet lag, shift work, and blindness, J. Biol. Rhythms 12 (1997) 604-617.

[5] Asplund R., Aberg H., Wetterberg L., The seasonal interrelationship between melatonin, vasopressin, and serum osmolality in elderly subjects, J. Pineal Res. 25 (1998) 67-72.

[6] Barnes R.G., Forbes M.J., Arendt J., Shift type and season affect adaptation of the 6-sulphatoxymelatonin rhythm in offshore oil rig workers, Neurosci. Lett. 252 (1998) 179-182.
[7] Bergstrom W.H., Hakanson D.O., Melatonin: the dark force. Adv. Pediatr. 45 (1998) 91-106.

[8] Brainard G.C., Kavet R., Kheifets L.I., The relationship between electromagnetic field and light exposures to melatonin and breast cancer risk: a review of the relevant literature, J. Pineal Res. 26 (1999) 65-100.

[9] Brusco L.I., Marquez M., Cardinali D.P., Monozygotic twins with Alzheimer's disease treated with melatonin: Case report, J. Pineal Res. 25 (1998) 260-263.

[10] Bubenik G.A., Blask D.E., Brown G.M., Maestroni G.J., Pang S.F., Reiter R.J., Viswanathan M., Zisapel N., Prospects of the clinical utilization of melatonin, Biol. Signals Recept. 7 (1998) 195-219.

[11] Cajochen C., Krauchi K., Danilenko K.V., WirzJustice A., Evening administration of melatonin and bright light: interactions on the EEG during sleep and wakefulness, J. Sleep Res. 7 (1998) 145-157.

[12] Ciesla W., Can high maternal melatonin concentrations be responsible for inducing fetal pathologies, and can melatonin participate in immunohormonal homeostasis by determining prohormone convertase activity? Hypothesis and facts, Med. Hypotheses 51 (1998) 269-280.

[13] Costa A., Leston J.A., Cavallini A., Nappi G., Cluster headache and periodic affective illness: common chronobiological features, Funct. Neurol. 13 (1998) 263-272.

[14] Dolberg O.T., Hirschmann S., Grunhaus L., Melatonin for the treatment of sleep disturbances in major depressive disorder, Am. J. Psychiatry 155 (1998) 1119-1121.

[15] Dreher F., Gabard B., Schwindt D.A., Maibach H.I., Topical melatonin in combination with vitamins $E$ and $C$ protects skin from ultraviolet-induced erythema: a human study in vivo, Br. J. Dermatol. 139 (1998) 332-339.

[16] Fjaerli O., Lund T., Osterud B., The effect of melatonin on cellular activation processes in human blood, J. Pineal Res. 26 (1999) 50-55.

[17] Garcia-Maurino S., Gonzalez-Haba M.G., Calvo J.R., Goberna R., Guerrero J.M., Involvement of nuclear binding sites for melatonin in the regulation of IL-2 and IL-6 production by human blood mononuclear cells, J. Neuroimmunol. 92 (1998) 76-84.

[18] Gubitz A.K., Reppert S.M., Assignment of the melatonin-related receptor to human chromosome X (GPR50) and mouse chromosome X, Genomics 55 (1999) 248-251.

[19] Jean-Louis G., von Gizycki H., Zizi F., Melatonin effects on sleep, mood, and cognition in elderly with mild cognitive impairment, J. Pineal Res. 25 (1998) 177-183.

[20] Kadva A, Djahanbakhch O., Monson J., Di WL., Silman R., Elevated nocturnal melatonin is a consequence of gonadotropin-releasing hormone 
deficiency in women with hypothalamic amenorrhea, J. Clin. Endocrinol. Metab. 83 (1998) 3653-3662.

[21] Karasek M., Woldanska-Okonska M., Czemicki J., Zylinska K., Swietoslawski J., Chronic exposure to $2.9 \mathrm{mT}, 40 \mathrm{~Hz}$ magnetic field reduces melatonin concentrations in humans, J. Pineal Res. 25 (1998) 240-244.

[22] Kendler B.S., Melatonin: media hype or therapeutic breakthrough?, Nurse Pract. 22 (1997) 66-77.

[23] Kunz D., Bes F., Schlattmann P., Hermann W.M., On pineal calcification and its relation to subjective sleep perception: a hypothesis-driven pilot study, Psychiatry Res. 30 (1998) 187-191.

[24] Lasko T.A., Kripke D.F., Elliot J.A., Melatonin suppression by illumination of upper and lower visual fields, J. Biol. Rhythms 14 (1999) 122-125.

[25] Lewy A.J., The dim light melatonin onset, melatonin assays and biological rhythm research in humans, Biol. Signals Pecept. 8 (1999) 79-83.

[26] Lewy A.J., Ahmed S., Latham-Jackson J.M., Sack R.L., Melatonin shifts human circadian rhythms according to a phase-response curve, Chronobiol. Int. 9 (1992) 380-392.

[27] Lewy A.J., Bauer V.K., Cutler N.L., Sack R.L., Ahmed S., Thomas K.H., Blood M.L., Jackson J.M., Morning vs evening light treatment of patients with winter depression, Arch. Gen. Psychiatry 55 (1998) 890-896.

[28] Li X., Borjigin J., Snyder S.H., Molecular rhythms in the pineal gland, Curr. Opin. Neurobiol. 8 (1998) 648-651.

[29] Liu R.Y., Zhou J.N., van Heerikhuize J., Hofman M.A., Swaab D.F., Decreased melatonin levels in postmortem cerebrospinal fluid in relation to ageing, Alzheimer's disease, and apolipoprotein E-epsilon4/4 genotype, J. Clin. Endocrinol. Metab. 84 (1999) 323-327.

[30] Lockley S.W., Skene D.J., Thapan K., English J., Ribeiro D., Haimov I., Hampton S., Middleton B., von Schantz M., Arendt J., Extraocular light exposure does not suppress plasma melatonin in humans, J. Clin. Endocrinol. Metab. 83 (1998) 3369-3372.

[31] Luboshitzky R., Yanai D., Shen-Orr Z., Israeli E., Herer P., Lavie P., Daily and seasonal variations in the concentration of melatonin in the human pineal gland, Brain Res. Bull. 47 (1998) 271-276.

[32] Mayeda A., Mannon S., Hofstetter J., Adkins M., Baker R., Hu K., Nurnberger J. Jr, Effects of indirect light and propranolol on melatonin levels in normal human subjects, Psychiatry Res. 81 (1998) 9-17.

[33] Miles A., Philbrick D.R., Melatonin and psychiatry, Biol. Psychiatry 23 (1998) 405-425.

[34] Millet B., Touitou Y., Poirier M.F., Bourdel M.C., Hantouche E., Bogdan A., Olie J.P., Plasma melatonin and cortisol in patients with obses- sive-compulsive disorder: relationship with axillary temperature, physical activity, and clinical symptoms, Biol. Psychiatry 44 (1998) 874-881.

[35] Monteleone P., Natale M., La Rocca A., Maj M., Decreased nocturnal secretion of melatonin in drug-free schizophrenics: no change after subchronic treatment with antipsychotics, Neuropsychobiology 36 (1997) 159-163.

[36] Mullen P.E., Silman R.E., The pineal and psychiatry: a review, Psychol. Med. 7 (1997) 407-417.

[37] Nishimura S., Fujino Y., Shimaoka M., Hagihira S., Taenaka N., Yoshiya I., Circadian secretion patterns of melatonin after major surgery, J. Pineal Res. 25 (1998) 73-77.

[38] Nonno R., Fraschini F., Stankov B.M., Methods for the evaluation of drug action at the human melatonin receptor subtypes, Biol. Signals Recept. 8 (1999) 32-40.

[39] Palm L., Blennow G., Wetterberg L., Long-term melatonin treatment in blind children and young adults with circadian sleep-wake disturbances, Dev. Med. Child Neurol. 39 (1997) 319-325.

[40] Partonen T., Extrapineal melatonin and exogenous serotonin in seasonal affective disorder, Med. Hypotheses 51 (1998) 441-442.

[41] Pillar G., Etzioni A., Shahar E., Lavie P., Melatonin treatment in an institutionalised child with psychomotor retardation and an irregular sleepwake pattern, Arch. Dis. Child 79 (1998) 63-64.

[42] Poirier M.F., Current trends in clinical psychiatric research, Presse Med. 19 (1998) 2185-2189.

[43] Rasmussen D.D., Boldt B.M., Wilkinson C.W., Yellon S.M., Matsumoto A.M., Daily melatonin administration at middle age suppresses male rat visceral fat, plasma leptin, and plasma insulin to youthful levels, Endocrinology 140 (1999) 1009-1012.

[44] Reber A., Huber P.R., Ummenhofer W., Gurtler C.M., Zurschmiede C., Drewe J., Schneider M., General anaesthesia for surgery can influence circulating melatonin during daylight hours, Acta Anaesthesiol. Scand. 42 (1998) 1050-1056.

[45] Reiter R.J., Oxidative damage in the central nervous system: protection by melatonin, Prog. Neurobiol. 56 (1998) 359-384.

[46] Reiter R.J., Maestroni G.J., Melatonin in relation to the antioxidative defense and immune systems: possible implications for cell and organ transplantation, J. Mol. Med. 77 (1999) 36-39.

[47] Rodenbeck A., Huether G., Ruther E., Hajak G., Altered circadian melatonin secretion patterns in relation to sleep in patients with chronic sleepwake rhythm disorders, J. Pineal Res. 25 (1998) 201-210.

[48] Shafii M., Shafii S.L., Melatonin in Psychiatric and Neoplastic Disorders, Am. Psych. Press, Inc., Washington, 1998.

[49] Slotten H.A., Krekling S., Does melatonin have an effect on cognitive performance?, Psychoneuroendocrinology 21 (1996) 673-680. 
[50] Stevens R.G., Wilson B.W., Anderson L.E., The Melatonin Hypothesis; Breast Cancer and the Use of Electric Power, Battelle Press, Columbus, 1997

[51] Suhner A., Schlagenhauf P., Johnson R., Tschopp A., Steffen R., Comparative study to determine the optimal melatonin dosage form for the alleviation of jet lag, Chronobiol. Int. 15 (1998) 655-666.

[52] Suhner A., Schlagenhauf P., Tschopp A., HauriBionda R., Friedrich-Koch A., Steffen R., Impact of melatonin on driving performance, J. Travel Med. 5 (1998) 7-13.

[53] Tan D.X., Manchester L.C., Reiter R.J., Plummer B.F., Hardies L.J., Weintraub S.T., Vijayalaxmi., Shepherd A.M., A novel melatonin metabolite, cyclic 3-hydroxymelatonin: a biomarker of in vivo hydroxyl radical generation, Biochem. Biophys. Res. Commun. 253 (1998) 614-620.

[54] Thalén B.E., Kjellman B.F., Morkrid L., Wetterberg L., Melatonin in light treatment of patients with seasonal and nonseasonal depression, Acta Psychiatr. Scand. 92 (1995) 274-284.

[55] Thalén B.E., Morkrid L., Kjellman B.F., Wetterberg L., Cortisol in light treatment of seasonal and non-seasonal depression: relationship between melatonin and cortisol, Acta Psychiatr. Scand. 96 (1997) 385-394.

[56] Thomas L., Drew J.E., Abramovich D.R., Williams L.M., The role of melatonin in the human fetus (review), Int. J. Mol. Med. I (1998) 539-543.

[57] Touitou Y., Melatonin: hormone and medication, C. R. Seances Soc. Biol. Fil. 192 (1998) 643-657.

[58] Van Cauter E., Putative roles of melatonin in glucose regulation, Therapie 53 (1998) 467-472.

[59] Visser E.K., Beersma D.G., Daan S., Melatonin suppression by light in humans is maximal when the nasal part of the retina is illuminated, J. Biol. Rhythms 14 (1999) 116-121.

[60] Vondrasova-Jelinkova D., Hajek I., Illnerova $H$., Adjustment of the human melatonin and cor- tisol rhythms to shortening of the natural summer photoperiod, Brain Res. 816 (1999) 249-253.

[61] Wahlund B., Grahn H., Saaf J., Wetterberg L., Affective disorder subtyped by psychomotor symptoms, monoamine oxidase, melatonin and cortisol: identification of patients with latent bipolar disorder, Eur. Arch. Psychiatry Clin. Neurosci. 248 (1998) 215-224.

[62] Wahlund B., Saaf J., Grahn H., Wetterberg L. Diagnostic subgrouping of depressed patients by principal component analysis and visualized pattern recognition, Psychiatry Res. 81 (1998) 393-401.

[63] Waldhauser F., Kovacs J., Reiter E., Age-related changes in melatonin levels in humans and its potential consequences for sleep disorders, Exp. Gerontol. 33 (1998) 759-772.

[64] Webb S.M., Puig-Domingo M., Moller M. Pévet P., Pineal Update; From Molecular Mechanisms to Clinical Implications, PJD Publications Ltd, Westbury, NY, USA, 1997.

[65) Wetterberg L., Melatonin and affective disorders, Ciba Found. Symp. 117 (1985) 253-265.

[66] Wetterberg L., Bergiannaki J.D., Paparrigopoulos T., von Knorring L., Eberhard G., Bratlid T., Yuwiler A., Normative melatonin excretion: a multinational study, Psychoneuroendocrinology 24 (1999) 209-226.

[67] Weydahl A., Sothern R.B., Wetterberg L., Seasonal variations in melatonin may modulate glycemic response to exercise, Percept. Mot. Skills 86 (1998) 1061-1062.

[68] Wikner J., Svanborg E., Wetterberg L., Rojdmark S., Melatonin secretion and excretion in patients with obstructive sleep apnea syndrome, Sleep 20 (1997) 1002-1007.

[69] Wikner J., Wetterberg L., Rojdmark S. , Does hypercalcaemia or calcium antagonism affect human melatonin secretion or renal excretion?, Eur. J. Clin. Invest. 27 (1997) 374-379.

[70] Wikner J., Hirsch U., Wetterberg L., Rojdmark S., Fibromyalgia - a syndrome associated with decreased nocturnal melatonin secretion, Clin. Endocrinol. (Oxf) 49 (1998) 179-183 